\title{
Combinando Técnicas de Mineração de Dados para Melhorar a Detecção de Indicadores de Evasão Universitária*
}

\author{
Davi Carrano, Elisa Tuler, Carlos Infante, Leonardo Rocha \\ Universidade Federal de São João del-Rei (UFSJ)- MG - Brazil \\ \{davi, etuler,prof.eduinfante, lcrocha\}@ufsj.edu.br
}

\begin{abstract}
The student' dropout is one of the major problems faced by public universities in Brazil since generates financial, social and academic loss. In this work, we present a methodology which combines different data mining techniques that aims: (1) creating predictive models to identify students who are at risk of dropout; and (2) establishing the relevance of the attributes related to the phenomenon and thus contribute to its prevention. We evaluate the methodology applying it in real information regarding students of the Federal University of São João Del Rei, performing both a global analysis of the data and a fragmented analysis for each Knowledge Area. The predictive models generated show alarming numbers for possible evasions in 2019, especially in Engineering; Agrarian Sciences; Exact and Earth Sciences; and Applied Social Sciences. Regarding the identified attributes, the academic indicators were the most relevant to understanding the student's dropout, a factor that places the institutional manager as the main figure to combat this phenomenon.
\end{abstract}

Resumo. A evasão estudantil é um dos maiores problemas enfrentados pelas universidades públicas brasileiras, pois gera perdas financeiras, sociais $e$ acadêmicas. Neste trabalho apresentamos uma metodologia que combina diferentes técnicas de mineração de dados com o objetivo de: (1) criar modelos preditivos para identificar alunos que se encontram em risco de evasão; e (2) estabelecer a relevância dos atributos relacionados ao fenômeno e assim contribuir com a prevenção do mesmo. Avaliamos a metodologia com dados reais de discentes da Universidade Federal de São João del-Rei realizando uma análise global dos dados e uma análise fragmentada para cada Área de Conhecimento. Os modelos preditivos gerados apresentam números alarmantes quanto às possíveis evasões em 2019, sobretudo nas Engenharias, Ciências Agrárias, Ciências Exatas e da Terra e Ciências Sociais Aplicadas. Com relação aos atributos identificados, os indicadores acadêmicos foram os mais relevantes para a compreensão do comportamento evasivo dos alunos, fator que coloca o gestor institucional como a figura principal para combater esse fenômeno.

\section{Introdução}

O problema de evasão universitária tem gerado prejuízos financeiros, sociais e acadêmicos, tanto para as Instituições Federais de Ensino Superior (IFES) quanto para os alunos [Da Cunha et al. 2016]. Os investimentos recebidos a partir das políticas de expansão universitária recentes foram altos e, entretanto, o impacto no quantitativo de egressos não têm sido proporcional a esse investimento. Apesar de se tratar de um fenômeno

${ }^{*}$ Esse trabalho foi parcialmente financiado por CNPq, CAPES, e Fapemig. 
VIII Congresso Brasileiro de Informática na Educação (CBIE 2019)

Anais do XXX Simpósio Brasileiro de Informática na Educação (SBIE 2019)

internacional [Dekker et al. 2009, Kotsiantis et al. 2003], a evasão universitária no Brasil é um problema público, pois afeta as decisões sobre investimentos do governo federal.

Há na literatura estudos dedicados à compreensão desse fenômeno visando diagnosticar os motivos que levam um aluno a abandonar uma universidade [Dekker et al. 2009, Kotsiantis et al. 2003, Sachin and Vijay 2012, Manhães et al. 2011, Da Cunha et al. 2016, Amorim et al. 2008, Oliveira Júnior et al. 2014]. Muitos dos trabalhos recentes são baseados na utilização de técnicas de Mineração de Dados em dados relacionados à trajetória acadêmica dos estudantes (linha de pesquisa conhecida como Mineração de Dados Educacionais). Mineração de Dados constitui-se de um conjunto de técnicas de inteligência artificial, teoria de grafos, banco de dados e conhecimentos de estatística com objetivo de encontrar informação útil a partir de grandes volumes de dados. Na detecção de evasão, alguns trabalhos estão focados na utilização de um subconjunto de técnicas relacionadas à Aprendizagem de Máquina (AM). Essas técnicas visam, a partir de certo conjunto de treino contendo informações relacionadas a estudantes que ainda se encontram na universidade, bem como estudantes egressos e que evadiram, criar um modelo preditivo para que um comportamento evasivo seja detectado tão logo se possa.

O presente trabalho propõe não somente a criação destes modelos preditivos que identificam os alunos em risco de evasão, mas também uma metodologia que utiliza diversas técnicas de mineração de dados, com o intuito de identificar os atributos mais relevantes que fizeram parte da formulação destes modelos, e com isto compreender o comportamento evasivo dos alunos, auxiliando assim na prevenção desse fenômeno. Nossa hipótese é que a utilização coordenada de diversas técnicas de mineração de dados pode auxiliar na compreensão dos dados relacionados à trajetória acadêmica dos estudantes e, assim, identificar de fato quais são os indicadores mais relevantes em relação ao comportamento evasivo. Identificar e compreender esses indicadores pode subsidiar e direcionar a elaboração de políticas nacionais de combate à evasão. Além disso, determinar a relevância desses indicadores pode melhorar os modelos preditivos, uma vez que as técnicas de AM podem focar nesses atributos mais relevantes [Sandin et al. 2012].

Avaliamos a metodologia utilizando dados reais da Universidade Federal de São João del-Rei (UFSJ), selecionando os dados pessoais, socioeconômicos e acadêmicos dos alunos de graduação presencial da instituição, entre os anos de 2010 e 2017. Realizamos tanto uma análise global sobre todo conjunto de dados, como também uma análise fragmentada para cada Área de Conhecimento. Os resultados deste estudo apresentam números alarmantes sobre a previsão de evasões na UFSJ para 2019, que afetam principalmente a área de conhecimento das Engenharias, Ciências Agrárias, Ciências Exatas e da Terra e Ciências Sociais Aplicadas. Os resultados também demonstram que os indicadores acadêmicos foram os mais relevantes para a compreensão desse comportamento evasivo, seja na análise da instituição como um todo ou de cada área de conhecimento. Dentro destes indicadores acadêmicos estão atributos relacionados ao desempenho dos alunos, à assiduidade e à satisfação. A interpretação destes atributos mais relevantes remete a um fracasso acadêmico, que é a impossibilidade do aluno em lidar com as dificuldades do sistema acadêmico e uma falha deste sistema em proporcionar as condições necessárias para que o aluno consiga isso. Esta interpretação coloca o gestor institucional como a figura principal no confronto ao problema da evasão universitária na UFSJ. 
VIII Congresso Brasileiro de Informática na Educação (CBIE 2019)

Anais do XXX Simpósio Brasileiro de Informática na Educação (SBIE 2019)

\section{Mineração de Dados Educacionais}

A área que utiliza técnicas de Mineração de Dados com o objetivo de analisar e compreender as informações relacionadas a dados educacionais recebe o nome de Educational Data Mining (EDM) - Mineração de Dados Educacionais. Trabalhos desenvolvidos nesta área visam auxiliar na resolução de questões importantes relacionadas à pesquisa educacional [Sachin and Vijay 2012], sendo a evasão de estudantes um dos maiores problemas abordados por tais técnicas. Vários trabalhos, tanto no cenário internacional quanto no cenário nacional, foram realizados utilizando a EDM para uma investigação do fenômeno da evasão. No cenário internacional, [Dekker et al. 2009] analisou dados dos alunos de graduação do curso presencial de Engenharia Elétrica da Eindhoven University of Technology, avaliando-se diferentes técnicas de classificação automática da ferramenta Weka. Outro trabalho nesta mesma linha foi apresentado em [Kotsiantis et al. 2003]. Utilizando dados de alunos de um curso de informática à distância da Hellenic Open University (HOU), os autores também avaliaram diversos algoritmos de classificação automática. O objetivo principal era criar uma ferramenta para auxiliar na identificação de alunos com risco de evasão já antes do meio do primeiro módulo do curso.

No cenário nacional, o trabalho de [Manhães et al. 2011] analisou dados dos alunos de graduação do curso presencial de Engenharia da Escola Politécnica da UFRJ, visando identificar precocemente aqueles que apresentavam risco de evasão. Os autores compararam vários algoritmos de classificação da ferramenta de mineração de dados $W_{e k a}{ }^{1}$. O trabalho de [Amorim et al. 2008], realizado na Universidade Federal do Rio Grande do Sul (UFRGS), demonstrou a aplicação de três algoritmos classificadores sobre a base de dados de uma instituição de ensino superior particular, base essa que representava 10 semestres letivos. Por fim, o trabalho de [Oliveira Júnior et al. 2014], realizado na UTFPR, demonstrou a aplicação de cinco algoritmos classificadores sobre a base de dados da própria UTFPR, base esta referente aos alunos que ingressaram no primeiro semestre de 2012, acumulando dados de 4 semestres consecutivos (de 2012/1 a 2013/2).

De forma geral, muitos trabalhos focam na aplicação e comparação de vários algoritmos de mineração de dados que geram modelos que visam prever alunos em risco de evasão. Um primeiro ponto que nos diferencia desses trabalhos se refere ao produto final dos mesmos que, basicamente, são os modelos preditivos, sem levar em consideração os fatores que mais influenciaram na previsão. A não identificação desses fatores dificulta ou até mesmo impossibilita o estabelecimento de políticas públicas educacionais para o combate do fenômeno. $\mathrm{O}$ segundo ponto se refere à análise da metodologia. Enquanto os trabalhos apresentados na literatura se concentram em uma análise baseada em dados de alunos de apenas um curso, a metodologia que propomos é aplicada aos dados de todos os alunos da graduação presencial da UFSJ, em dois cenários diferentes: (1) envolvendo todos os cursos, para uma análise da evasão na instituição como um todo; e (2) sobre cada Área de Conhecimento em separado. O conhecimento do fenômeno para cada área em separado visa contribuir para o direcionamento das políticas públicas educacionais por nicho de conhecimento. Por fim, outro diferencial do nosso trabalho está no recorte temporal dos dados, que envolve alunos que ingressaram entre os anos de 2010 e 2017, em detrimento aos recortes da literatura, que contemplam pequenos espaços temporais.

\footnotetext{
${ }^{1}$ https://www.cs.waikato.ac.nz/ml/weka/
} 
VIII Congresso Brasileiro de Informática na Educação (CBIE 2019)

Anais do XXX Simpósio Brasileiro de Informática na Educação (SBIE 2019)

\section{Metodologia}

Nessa seção apresentamos a principal contribuição desse trabalho: uma metodologia que combina de forma coordenada diferentes técnicas de mineração de dados com objetivo de gerar modelos preditivos que melhor identifiquem estudantes propensos a evadir, bem como inferir, dentre os atributos pessoais, socioeconômicos e acadêmicos dos estudantes, aqueles mais fortemente associados a esse fenômeno. Nossa metodologia é constituída de 6 etapas principais descritas a seguir e devidamente sumarizadas na Figura 1.

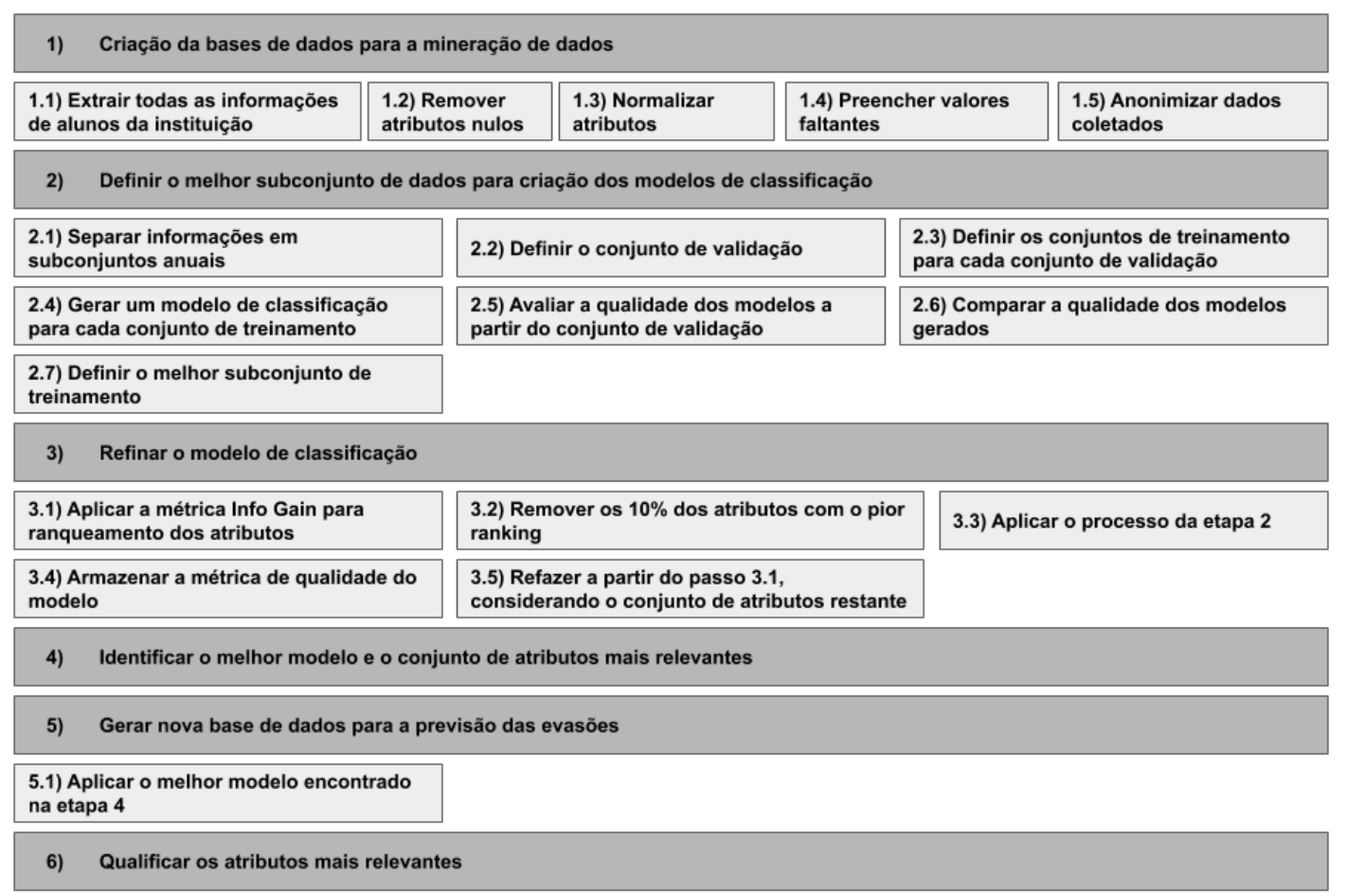

Figura 1. Metodologia para identificar os indicadores de evasão universitária.

1. A primeira etapa consiste na criação da base de dados que será utilizada em todas as etapas seguintes. Para isso, devemos extrair todas as informações dos estudantes da instituição, sejam informações pessoais, tais como cidade e estado de nascimento, idade, sexo, satisfação com curso, etc., socioeconômicas tais como tipo da vaga (tipo de cota ou ampla concorrência), tipo de admissão (SISU, vestibular, transferência, etc.), situação financeira, acesso à tecnologias, etc. e acadêmicos tais como notas e frequência em unidades curriculares, avaliação dada aos docentes das unidades curriculares cursadas, dentre outras. A meta dessa etapa é extrair o máximo de informações possíveis, sem nenhum tipo de prejulgamento da importância dos atributo. Faz parte dessa etapa a aplicação de estratégias de pré-processamento, tais como remoção de atributos nulos, normalização de atributos, preenchimento de valores faltantes, anonimização, etc.

2. O segundo passo da metodologia é definir qual o melhor subconjuntos de dados para ser utilizado na criação dos modelos preditivos. Conforme vimos na seção anterior, grande parte dos trabalhos voltados à previsão de evasão, criam seus modelos considerando todas as informações de todo o período dos dados coletados. Consideramos a hipótese que, dados referentes à um passado longínquo podem apresentar distorções quanto aos dados mais atuais [Mourão et al. 2008]. 
Assim, essa etapa consiste em definir qual a porção de dados do passado deve ser considerada na geração do modelo de predição. Considerando que a base de dados construída na etapa anterior conterá informações referentes a diversos anos, a primeira tarefa dessa etapa consiste em separar as informações em diversos subcojuntos por ano. A partir disso, devemos fixar os dados referentes a um determinado ano como nosso conjunto de validação, ou seja, aquele conjunto de dados para os quais já sabemos o classe do aluno ("Evadiu" ou "Não Evadiu") e que deverá ser avaliado pelo modelo preditivo criado a partir de um algoritmo de classificação (e.g. árvore de decisão, Naïve Bayes, SVM, etc.), utilizando como treinamento os dados referentes aos demais anos anteriores. A proposta é variar esse conjunto de treinamento considerando dados de: 1 ano anterior; 2 anos anteriores; 3 anos anteriores; e 4 anos anteriores. Para cada conjunto de treinamento utilizado, devemos avaliar a qualidade do modelo gerado (e.g. Macro $F 1$, Acurácia, etc.) na predição das classes do conjunto de validação.

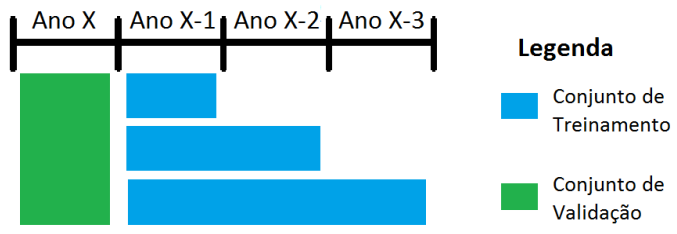

Figura 2. Método de elaboração dos conjuntos de treinamento e validação.

Na Figura 2 ilustramos esse processo. Por exemplo, escolhemos o Ano $X$ como nosso alvo (validação) e criamos um modelo utilizando os dados referentes apenas ao Ano $X-1$. Avaliamos a qualidade do modelo gerado classificando os dados do Ano $X$, em que a classe estimada pelo classificador (i.e. evasão ou não evasão) é comparada com a classe real do aluno. Repetimos esse processo considerando os dados do Ano $X-1$ e do Ano $X-2$ para gerar o modelo avaliando-o no Ano $X$, em seguinda utilizamos os dados do Ano $X-1$, do Ano $X-2$ e do Ano $X-3$ novamente avaliando-o no Ano $X$. Comparamos a qualidade dos modelos e o melhor subconjunto de dados de treinamento é então considerado nas etapas seguintes. Todo esse processo deve seguir a metodologia de avaliação hold-out de 80-20 com 50 repetições [Sandin et al. 2012] para que as comparações possam ser validadas estatisticamente. Qualquer algoritmo de classificação automática pode ser instanciado nessa etapa da metodologia, entretanto pela simplicidade e bom desempenho, sugerimos os algoritmos baseados em árvore de decisão.

3. A terceira etapa consiste em refinar o modelo de classificação gerado na etapa anterior combinando o algoritmo de classificação com uma métrica de seleção de características. Técnicas de seleção de características são capazes de associar um valor a cada um dos atributos de uma coleção de dados que corresponde à sua importância no processo de geração do modelo de classificação. Existem diversas métricas propostas na literatura [Sandin et al. 2012], baseadas em diferentes abordagens. Nossa sugestão é a adoção da métrica Info Gain [Sandin et al. 2012], por sua simplicidade, pelos bons resultados reportados na literatura e por ser genérica em termos de cenário de aplicação. Assim, primeiramente aplica-se a métrica na coleção de dados para que um valor de importância seja associado a cada um dos atributos. A partir desse valor, os atributos devem ser ordenados de forma crescente, ou seja, dos atributos de menor valor associado (menor 
VIII Congresso Brasileiro de Informática na Educação (CBIE 2019)

Anais do XXX Simpósio Brasileiro de Informática na Educação (SBIE 2019)

importância no processo de classificação) para os de maior valor. A partir disso, adota-se os seguintes passos:

(a) remove-se da coleção original os X\% de atributos de menor valor;

(b) aplica-se o processo descrito na etapa 2 com a base resultante da etapa 3(a), armazenando a qualidade do modelo gerado a partir dessa coleção.

Esse processo é repetido variando-se o valor de $X$ entre $10 \%, 20 \%, 30 \%, 40 \%$, $50 \%, 60 \%, 70 \%, 80 \%, 90 \%$, sempre guardando a qualidade dos modelos gerados em cada uma das etapas. A partir da avaliação da qualidade de cada modelo de classificação gerado é possível identificar aquele subconjunto de atributos de maior relevância para auxiliar na criação do melhor modelo de classificação.

4. A quarta fase consiste em comparar a qualidade do modelo de classificação gerados na etapa 2 (considerando todos os atributos extraídos na etapa 1) com cada um dos modelos de classificação gerados a partir do refinamento realizados na etapa 3 da metodologia (modelos que consideram apenas os atributos mais relevantes). Dentre todos os modelos, aquele que apresenta a melhor qualidade (com maior taxa de acerto e menor taxa de erro) será utilizado nas etapas seguintes.

5. Utilizando o mesmo processo descrito na etapa 1, gera-se uma nova coleção contendo os dados dos alunos ingressantes no ano atual (i.e. 2019). Nessa fase, aplicamos o melhor modelo encontrado na etapa 4 nessa nova coleção para se realizar uma nova avaliação do mesmo. Esse será o modelo que poderá ser utilizado sempre pelos órgãos competentes da IFES para realizar algum tipo de ação preventiva junto aos alunos considerados vulneráveis com relação à evasão.

6. A sexta e última etapa consiste em qualificar quais os atributos mais relevantes no processo de classificação, ou seja, quais atributos receberam os mais altos valores de importância pelo método de seleção de características. Essa é a etapa que mais nos distingue dos demais trabalhos propostos na literatura. A ideia é avaliar qualitativamente quem são esses atributos e o quão importante são os mesmos para se inferir a evasão ou não de um estudante. Ao final dessa etapa, teremos definido os atributos mais importantes relacionados à evasão dos estudantes na instituição. A partir dessa etapa a IFES será capaz de não só identificar quem são os alunos propensos a evadir, mas também conhecer os motivos que podem levá-lo a tomar essa atitude e, assim, promover ações preventivas no combate à evasão.

Por fim, é importante ressaltar que toda a metodologia acima pode ser aplicada considerando todos os dados da instituição, bem como separadas por área de conhecimento ou até mesmo por curso. Conforme veremos na seção seguinte, avaliamos a metodologia utilizando dados reais da Universidade Federal de São João del-Rei (UFSJ), selecionando os dados dos alunos de graduação presencial da instituição, entre os anos de 2010 e 2017. Realizamos tanto uma análise global sobre todo o conjunto de dados, como também uma análise fragmentada para cada Área de Conhecimento.

\section{Avaliando a Metodologia}

\subsection{Coleção de Dados e Ferramenta de Mineração de Dados}

A coleção de dados é constituída por 15.135 alunos de graduação presencial da UFSJ, que ingressaram no $1^{\circ}$ semestre entre os anos de 2010 e 2017. Selecionamos 77 atributos, dos quais 7 são de informações pessoais, 31 de informações acadêmicas e 39 de informações 
VIII Congresso Brasileiro de Informática na Educação (CBIE 2019)

Anais do XXX Simpósio Brasileiro de Informática na Educação (SBIE 2019)

socioeconômicas. Aplicamos as estratégias de pré-processamento discutidas na Etapa 1 da metodologia. A ferramenta de mineração de dados utilizada foi o R-Studioº ${ }^{2}$

\subsection{Definição do subconjunto de dados para criação dos modelos de classificação}

Neste tópico, são apresentados os resultado das Etapas 2, 3 e 4 da metodologia proposta. Inicialmente, as informações dos vários anos de análise foram separadas, formando 7 subconjuntos de validação (2011-2017), utilizados para a avaliação dos modelos preditivos, e 28 subconjuntos distintos de treinamento, utilizados para a criação dos modelos preditivos a partir do algoritmos de árvore de decisão. A Figura 3 ilustra esses subconjuntos.

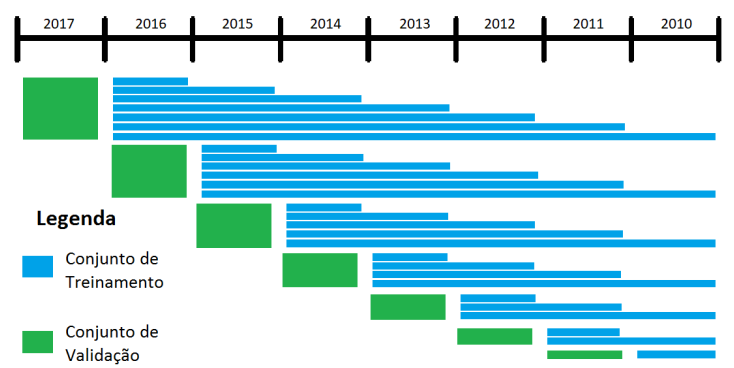

Figura 3. Subconjuntos de treinamento e validação elaborados.

Avaliamos cada conjunto de validação com cada uma das possibilidades de conjunto de treinamento. Por exemplo, considerando como validação o ano de 2013, consideramos os modelos criados a partir dos dados de: (1) 2012; (2) 2012 e 2011; e (3) 2012, 2011 e 2010. Além disso, cada um dos modelos preditivos foram gerados considerando todas os atributos, bem como uma variação em cima dos atributos mais importantes segundo a métrica Info Gain, conforme descrito na Etapa 3 da metodologia. Os melhores resultados encontrados foram para aqueles modelos que consideravam apenas $20 \%$ dos atributos mais relevantes. Na Tabela 1 apresentamos uma sumarização dos resultados desses experimentos. Para o conjunto de cada ano, apresentamos os resultados de qualidade, medidos por meio da área da curva ROC [Sandin et al. 2012], considerando cada um dos possíveis conjuntos de treinamento. Os resultados reportados já são aqueles considerando apenas os $20 \%$ de atributos mais relevantes.Conforme podemos observar na Tabela 1, na grande maioria dos casos, os modelos gerados considerando como conjunto de treinamento apenas os dados referentes ao ano imediatamente anterior foram aqueles que apresentaram os melhores resultados. Trata-se, na verdade, do conjunto de dados que oferece o melhor compromisso entre eficácia (qualidade dos resultados) e eficiência (baixo custo computacional e baixa complexidade de pré-processamento). Uma exceção, nesse caso, está relacionado aos modelos gerados para classificar os dados de 2014. A explicação para esse fato é que a UFSJ aderiu, por completo, seu processo de seleção ao SISU a partir de 2014. Até esse ano, na inscrição para o processo seletivo para ingressar na UFSJ, os candidatos preenchiam um conjunto muito grande de dados socioeconômicos e que descreviam muito bem suas situações pré-universidade. Conforme podemos perceber, esses dados são muito relevantes na geração dos modelos que estimam a propensão de evasão dos estudantes. Portanto, conforme veremos na Seção 5, um dos nossos objetivos é coletar e incluir informações pré-universidade dos estudantes, considerando outras fontes externas à UFSJ, para melhorar ainda mais a

\footnotetext{
${ }^{2}$ https://www.rstudio.com/
} 
VIII Congresso Brasileiro de Informática na Educação (CBIE 2019)

Anais do XXX Simpósio Brasileiro de Informática na Educação (SBIE 2019)

qualidade dos modelos gerados. Todo o processo descrito nessa seção também foi realizado considerando os dados separados por cada uma das Áreas de Conhecimento. Destacamos que o comportamento dos modelos preditivos gerados foram muito parecidos com aqueles que consideravam todos os dados. Por uma questão de limitação de espaço, o detalhamento desses resultados foram omitidos nessa seção.

Tabela 1. Comparação da qualidade dos modelos de acordo com a área da curva ROC. $O$ símbolo - representa empates estatísticos, $\Delta$ representa ganhos estatísticos e $\nabla$ representa perdas estatísticas, todos em relação ao modelo que considera como treinamento os dados do ano anterior.

\begin{tabular}{|l|l|l|l|l|l|l|l|}
\cline { 2 - 8 } \multicolumn{1}{c|}{} & \multicolumn{7}{c|}{ Conjuntos de Validação } \\
\cline { 2 - 8 } \multicolumn{1}{c|}{} & $\mathbf{2 0 1 7}$ & $\mathbf{2 0 1 6}$ & $\mathbf{2 0 1 5}$ & $\mathbf{2 0 1 4}$ & $\mathbf{2 0 1 3}$ & $\mathbf{2 0 1 2}$ & $\mathbf{2 0 1 1}$ \\
\hline Treino 1 & 0,81 & 0,72 & 0,69 & 0,72 & 0,58 & 0,65 & 0,63 \\
\hline Treino 2 & $0,79 \mathbf{\nabla}$ & $0,71 \bullet$ & $0,69 \bullet$ & $0,69 \mathbf{\nabla}$ & $0,68 \boldsymbol{\Delta}$ & $0,68 \boldsymbol{\Delta}$ & \\
\hline Treino 3 & $0,78 \boldsymbol{\nabla}$ & $0,73 \bullet$ & $0,70 \bullet$ & $0,72 \bullet$ & $0,66 \boldsymbol{\Delta}$ & & \\
\hline Treino 4 & $0,78 \boldsymbol{\nabla}$ & $0,72 \bullet$ & $0,69 \bullet$ & $0,71 \bullet$ & & & \\
\hline Treino 5 & $0,78 \boldsymbol{\nabla}$ & $0,72 \bullet$ & $0,71 \boldsymbol{\Delta}$ & & & & \\
\hline Treino 6 & $0,77 \mathbf{\nabla}$ & $0,71 \bullet$ & & & & & \\
\hline Treino 7 & $0,76 \mathbf{\nabla}$ & & & & & & \\
\hline
\end{tabular}

\subsection{Previsão da evasão para o ano de 2019}

Nessa seção apresentamos os resultados relacionados à aplicação da Etapa 5 da metodologia. Conforme observado na seção anterior, os melhores modelos foram alcançados utilizando-se como treinamento apenas os dados referentes ao ano anterior, considerando apenas os $20 \%$ dos atributos mais relevantes. Sendo assim, geramos um modelo preditivo considerando os dados consolidados de 2018 para avaliar o modelo considerando os dados de alunos em 2019. Em nossas análises, consideramos apenas possíveis evasões que podem ocorrer nos quatro primeiros anos de curso. Assim, a base de dados a ser avaliada de 2019 constam alunos ingressantes em 2016, 2017 e 2018. É importante mencionar que a UFSJ ainda não tem os dados de 2019 consolidados no presente momento. Assim, as análises apresentadas a seguir são feitas com base na predição dada pelo modelo gerado. Todas essas análises estão fundamentadas no modelo gerado a partir de toda a coleção. Entretanto, resultados semelhantes foram encontrados para os modelos por Área de Conhecimento, mas por uma questão de limitação de espaço, não serão detalhados.

Considerando um total de mais de 4.000 estudantes ingressantes entre os anos de 2016 e 2018 ainda vinculados à UFSJ, temos que 10\% dos alunos ingressantes na UFSJ de 2016 à 2018 apresentam risco de evasão. 75\% desses alunos estão concentrados nas áreas de Engenharias, Ciências Agrárias, Ciências Exatas e da Terra e Ciências Sociais Aplicadas, sendo a primeira a área mais alarmante, $36 \%$ desse total. Trata-se de uma informação importante para o gestor da instituição que precisará tomar medidas de caráter reativo e imediato para tentar reverter esse quadro. Uma pergunta que surge a partir dessas análises é: Quais os principais fatores associados a esse alto índice de evasão? A resposta a essa pergunta permite ao gestor tomar medidas preventivas, atenuando esses fatores. Na seção seguinte respondemos essas questões.

\subsection{Qualificando os atributos mais relevantes no comportamento evasivo na UFSJ}

Nessa etapa nosso objetivo é analisar os atributos mais relevantes obtidos nos passos anteriores. Trata-se do principal diferencial de nosso trabalho frente aos demais apresentados na literatura [Sachin and Vijay 2012, Manhães et al. 2011]. Ordenamos os atributos de 
VIII Congresso Brasileiro de Informática na Educação (CBIE 2019)

Anais do XXX Simpósio Brasileiro de Informática na Educação (SBIE 2019)

forma decrescente em relação à sua relevância no processo de classificação, ou seja, dos atributos de maior relevância (maior valor de Info Gain) para os de menor relevância.

Primeiramente, focamos nossa análise nos atributos de maior relevância obtidos pelo modelo que considera todos os dados de 2018. Os atributos de maior Info Gain foram: média dos conceitos das avaliações que o aluno faz em relação ao docente e em relação a si próprio; coeficiente de rendimento dos alunos nos dois primeiros semestres; media de faltas dos alunos no primeiro ano; media de nota dos alunos no primeiro ano; volume de disciplinas cursadas; percentual de aprovação dos alunos no primeiro ano; desempenho do aluno nas 3 disciplinas com maior índice de reprovação em seus cursos. Diante desta relação de indicadores, a evasão na UFSJ pode ser compreendida ao se analisar atributos nas categorias de desempenho, assiduidade e satisfação. Esse fato propicia a interpretação de que as causas da evasão estão relacionadas com um fracasso acadêmico. Este fracasso diz respeito às dificuldades dos alunos em lidar com suas atribuições no ambiente acadêmico e admite uma parcela de responsabilidade por parte do sistema educacional, ao não proporcionar as condições necessárias para que os alunos consigam isto.

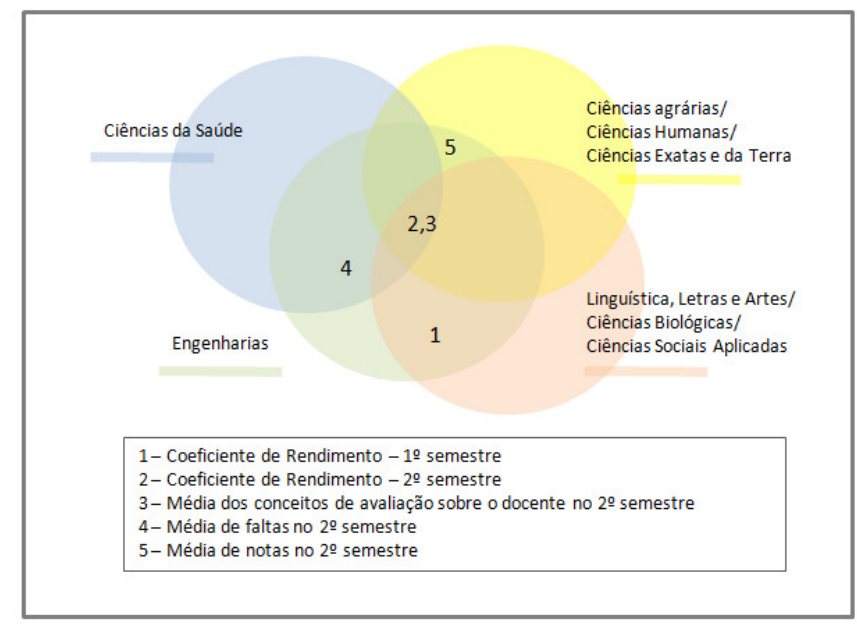

Figura 4. Diagrama de Venn - Indicadores de evasão por Área de Conhecimento.

Em um segundo momento, avaliamos os atributos mais relevantes pelos modelos gerados para cada Área de Conhecimento e os resultados podem ser visualizados pelo Digrama de Venn apresentado na Figura 4. A análise da evasão por Área de Conhecimento contribui para o direcionamento das políticas públicas educacionais por parte dos gestores institucionais. Na UFSJ, políticas voltadas para o combate ao fenômeno em questões relacionadas com o desempenho e a satisfação dos alunos devem ser direcionadas para todas as áreas de conhecimento. Já as políticas voltadas para as questões relacionadas com a assiduidade devem ser prioritariamente direcionadas para as áreas de Ciências da Saúde e Engenharias. Esses resultados corroboram estudos anteriores [Silva Filho et al. 2007] que ressaltam a importância de questões de ordem acadêmica como fontes de estímulo para o abandono ou a permanência. $\mathrm{O}$ avanço nas duas últimas décadas das políticas educacionais como o Programa Nacional de Assistência Estudantil (PNAES), voltadas para o público em situação de vulnerabilidade, contribuiu efetivamente para essa mudança de foco na motivação da evasão. Esses resultados destacam a necessidade do gestor institucional de prover ações tanto do ponto de vista pedagógico (i.e. ações para suprir a deficiência da formação em nível de Educação Básica) quanto do ponto de vista simbólico (i.e. açoes de integração dos novos estudantes às novas rotinas e novas culturas). 
VIII Congresso Brasileiro de Informática na Educação (CBIE 2019)

Anais do XXX Simpósio Brasileiro de Informática na Educação (SBIE 2019)

\section{Conclusões e Trabalhos Futuros}

Neste trabalho apresentamos uma metodologia que combina diferentes técnicas de mineração de dados com o objetivo de não apenas gerar modelos de classificação que auxiliem as gestões universitárias em identificar estudantes propensos à evasão, mas também de identificar quais são os atributos mais importantes relacionados a esse comportamento. Avaliamos a metodologia utilizando as informações de todos os alunos da graduação presencial da UFSJ, realizando uma análise global dos dados e por Área de Conhecimento. Em nossas análises identificamos que o desempenho dos estudantes, a satisfação e a assiduidade são categorias de informações fundamentais para determinar a evasão ou não, e refletem um tipo de fracasso acadêmico que elenca o gestor institucional como ator fundamental no processo de combate à evasão na instituição. Como trabalho futuro, nosso objetivo é coletar e incluir informações pré-universidade dos estudantes com o objetivo de criar modelos preditivos que sejam capazes de realizar previsões já no primeiro ano de curso. Pretender estender esse estudo também para os estudantes de cursos a distância.

\section{Referências}

Amorim, M. J. V., Barone, D., and Mansur, A. U. (2008). Técnicas de aprendizado de máquina aplicadas na previsão de evasão acadêmica. SBIE.

Da Cunha, J. V. A., Nascimento, E. M., and de Oliveira Durso, S. (2016). Razões e influências para a evasão universitária: um estudo com estudantes ingressantes nos cursos de ciências contábeis de instituições públicas federais da região sudeste. Advances in Scientific and Applied Accounting, 9(2):141-161.

Dekker, G., Pechenizkiy, M., and Vleeshouwers, J. (2009). Predicting students drop out: A case study. In Educational Data Mining 2009.

Kotsiantis, S. B., Pierrakeas, C., and Pintelas, P. E. (2003). Preventing student dropout in distance learning using machine learning techniques. In KES Conference. Springer.

Manhães, L. M. B., Da Cruz, S. M. S., Costa, R. J. M., Zavaleta, J., and Zimbrão, G. (2011). Previsão de estudantes com risco de evasão utilizando técnicas de mineração de dados. In SBIE, volume 1.

Mourão, F., Rocha, L., Araújo, R., Couto, T., Gonçalves, M., and Meira Jr., W. (2008). Understanding temporal aspects in document classification.

Oliveira Júnior, J. G., Noronha, R. V., and Kaestner, C. A. A. (2014). Análise da correlação da evasão de cursos de graduação com o empréstimo de livros em biblioteca. Anais dos Workshops do Congresso Brasileiro de Informática na Educação.

Sachin, R. B. and Vijay, M. S. (2012). A survey and future vision of data mining in educational field. In Advanced Computing \& Communication Technologies (ACCT), 2012 Second International Conference on, pages 96-100. IEEE.

Sandin, I., Andrade, G., Viegas, F., Madeira, D., Rocha, L., Salles, T., and Gonçalves, M. (2012). Aggressive and effective feature selection using genetic programming. In 2012 IEEE Congress on Evolutionary Computation, pages 1-8.

Silva Filho, R. L. L., Motejunas, P. R., Hipólito, O., and Melo Lobo, M. B. d. C. (2007). A evasão no ensino superior brasileiro. In Cadernos de Pesquisa, volume 37, pages 641-659. 